\title{
Implementation of Virtual Desktop Infrastructure in academic laboratories
}

\author{
Pawel Chrobak \\ Wroclaw, University of Economy \\ Komandorska 118-120, \\ 53-345 Wroclaw, Poland \\ Email: pawel.chrobak@ue.wroc.pl
}

\begin{abstract}
Abstrac-The article describes the causes of the economic and organizational case for implementing VDI solutions in the learning centers of Academic Centers.

The analysis laboratory infrastructure allows to better understand the broad ability to adapt to VDI and range of benefits they receive, administrators and staff research and teaching. Described implementation is based on VMware Horizon View 5.

The author was the originator of the concept of VDI implementation at the University of Economics in Wroclaw and participant of the project team.
\end{abstract}

\section{INTRODUCTION}

$\mathrm{T}$ HE increasing popularization of centralized computing centers popularly referred to as the clouds [5] caused the Universities of Poland to begin building their own private clouds, not only to support their internal processes, but also to provide the students with the virtualized workstation model DaS ( Desktop as a Service) [7].

The concept of DaS is to use a VDI environment to offer customers a persistent, highly available desktop that can be accessed from all of their mobile devices. This is significant because right now there are no services available that offer those services. The idea is to take the burden off of the customer by removing the tedious upkeep that the facilities must regularly go through. Desktop as a Service, or DaaS, is used in enterprises for a similar reason. The employees no longer have to worry about maintaining a PC, since the operating system would be centrally managed. The idea is to reduce complexity by centralizing management, which leads to a more productive and efficient IT organization.

This is significant because as the times change consumers are looking for different, innovative solutions to their problems. One problem that consumers have is that they have to regularly maintain their data and hardware. Consumer DaaS would provide a turnkey solution for consumers looking for a simpler computer experience [10].

The Article omits the description of Virtualization as it is generally known. Instead, after a brief description of the
VDI we will focus on the analysis of the suitability and potential benefits for virtualization of student laboratory.

The author is an employee of one of the first universities in Poland (University of Economics in Wroclaw) which implemented this solution on a massive scale in their teaching process. In this article, we describe some of the benefits and other experiences gained from the implementation of this project.

The subject of this study are student academic laboratories, although the content contained herein also refers to any type of training centers and educational solutions in schools or centers of learning.

\section{DESKTOP VIRTUALIZATION (VDI)}

For high end graphics workstations, consolidation seeks to migrate the processing load from multiple high end workstations to enterprise class servers in the data center. The primary goal is to offload the work from the local station and provide a desktop environment to users remotely. Rather than stack up a collection of workstations in the data center and provide remote access on a one-to-one basis, desktop virtualization utilizes virtualization techniques to consolidate multiple desktop workstations onto a single server. VMware, an industry leader in virtualization technologies, has created an alliance of vendors and service providers that support what they have coined the Virtual Desktop Infrastructure (VDI). Basically VDI is a server-based computing offering that provides desktop environments as an enterprise hosted service [19]. The core of the VDI initiative can be VMware's ESX server (or other virtualization solution) technology which provides hardware virtualization. Multiple separate operating system images and associated software packages share a single hardware server. Each instance is called a virtual machine (VM). For example, a VMware ESX server might host Microsoft Windows XP, Microsoft Windows Server family, Windows $7 / 8$, and Linux virtual machines at the same time. In its simplest form, each user can connect to a specific virtual machine using some kind of remote desktop protocol. However, having a 
dedicated virtual machine for each user is often impractical, unnecessary and cumbersome. Therefore, VDI solutions normally include some kind of connection broker to connect users to available VM's. Connection brokers are a part of a rapidly developing suite of management tools that can help minimize the support overhead of a VDI solution. Management tools may include services to connect users to the correct pool of VM's, determine which VM's are in use, locate active users, automatically reconnect disconnected remote sessions, provision additional VM's on demand, take VM's offline for testing, updating or troubleshooting, remotely relocate, reboot and reset running and offline VM's, anticipate performance issues or equipment failures, monitor performance, and perform load balancing. It is helpful to compare and contrast VDI with previous generations of remote desktop solutions. Server Based Computing (SBC) is one solution that has developed over the last 10 years, providing applications and desktops to users. Users connect to a remote server, sharing a single instance of an operating system and applications. The application access is increasingly seamless, providing users an illusion that they are working on the application locally even though it is executing on the remote server. Rather than each user getting a VM to themselves, users share connections to the server operating system and installed applications. Citrix Systems has coined the Dynamic Desktop Initiative (DDI) to contrast VMware's VDI. DDI is a Windows based desktop that's delivered over any network and optimized for office ... tasks - from simple to complex. DDI is a developing initiative that builds on current solutions. Desktop virtualization is a term that can apply to other types of virtualization strategies. For example, a desktop workstation can be utilizing a desktop virtualization product to allow several operating systems to run simultaneously on one local desktop machine. One common example of this type of desktop virtualization is in the software development life cycles, where it is helpful to have a virtualized production environment available to the developer immediately. This is particularly useful in software development test-bed scenarios. Other desktop virtualization strategies focus on getting a standardized application or operating system image out to local workstations, streaming applications or operating systems out to office computers or unsecured terminals. In these scenarios, the local workstation hardware runs the operating system and/or software that are being provided from a remote source. Note that this does not match our scenario. In both notebook computer desktop virtualization and high end graphic workstation consolidation, the applications and operating system will be running on the remote servers. VDI and DDI approaches each have their strengths and weaknesses. For instance, due to lower overhead, Citrix Presentation Server can support more users per server. However, the applications run in the server operating system environment, rather than the Windows XP professional as they could in a VDI solution. This poses some challenges for us because the applications our users employ are created for use in desktop level operating systems such as Windows XP.
They are often not well tested and qualified in the Windows Server environment. How would the developing VDI (VMware) and DDI (Citrix) solutions meet the desktop virtualization challenges presented by Ringling College? Only hands on testing would tell[8].

\section{GENERAL BENEFITS OF IMPLEMENTATION OF VDI SOLUTIONS}

Computer labs students are usually characterized by low utilization of resources at a fairly fast computer purchased to laboratories. The low utilization statistics indicate that workstation consolidation could achieve great savings in infrastructure, networking, power consumption, and maintenance costs. In addition, we would spend less time in deployment, security, and fault isolation without compromising performance.

With the ever increasing prices of upgrading desktop computers, virtualization of the desktop is becoming very appealing. Here are some of the benefits of virtual desktop infrastructure (VDI).

- Management - In a typical corporate infrastructure, you manage desktops using remote software technology such as Altiris or some other push technology. It is really hard to man-age hundreds of desktops as you are well aware if you administer desktops in your corporate infrastructure. Using technology such as virtual desktop infrastructure (VDI) allows you to have central management of all your desktops and really control what is being installed and used on the desktops. Deployment of virtual desktops is lightning fast as opposed to using imaging technology such as Norton or other antiquated technologies. Would you like to manage 500 desktops all over the United States or Europe or manage them from one data center?

- Security - Security is a key factor in rolling out VDI. With VDI, you have greater control of how you secure your desktop. You can lock down the image from external devices or pre-vent copying data from the image to your local machine; I'm a big fan of this feature of VDI. Remote users or road warriors also benefit as sensitive data is stored on the server in the data center and not the device. If the device is stolen, the information is protected.

- OS migrations - Let's say you want to roll out Windows Vista to a select few managers. Prior to VDI, you would have to look at their equipment and most likely upgrade hardware, memory, disk space, etc. With VDI, you can just push out a Windows Vista image from a central location to the group of managers.

- VDI image - We can create a library of VDI images to meet all of your company needs. If your company is seasonal, you can have extra images to handle the increased employee traffic. If you use third-party vendors/contractors/consultants, you can use secure/encrypted locked down images to allow them to work in your environment. 
- Snapshot technology - With VDI, you have the ability to roll back desktops to different states. This is a great feature, and it allows you to give a lot of flexibility to your end users.

- Go green - A thin client VDI session will use less electricity than a desktop computer. Using VDI is a way to reduce your carbon footprint on our planet and save your company money in power costs.

- Independence - With VDI, who cares what device you use? A thin client, a PC, Apple, Linux, etc. As long as you can connect to your VDI with ICA or RDP, you are golden[11].

\section{ECONOMIC AND ORGANIZATIONAL CAUSES OF IMPLEMENTATION OF VDI IN ACADEMIC ENVIRONMENT}

Universities have a few reasons for deploying VDI in teaching laboratories: economic, organizational, technological or marketing. In fact almost all aspects have also the implied economic savings (redundancies, etc.), so in the characteristics below will focus on the economic and organizational aspects.

The reality was that desktop support had focused on bringing broken machines back from the dead and not on discovering new tools or ways in which they could be used. Desktop and laptop computer users were supporting themselves in regard to using software and the number of trouble tickets handled clearly indicated that it wasn't about the user. IT wasn't supporting the user of the desktop but instead the computer that was on the desktop. To improve customer service and deliver better support, this paradigm had to change and the only way to change it was to substitute the need for hardware fixes with the ability to apply user fixes. Solving the problems that the user had required extending the knowledge base to them and eliminating the break fix endless loop that existed [9].

\section{V.CHARACTERISTICS OF STUDENT'S LABORATORIES}

Computer workstations in the student laboratories have their own characteristics, the analysis and understanding of them allow us to understand the ability to adapt to VDI in such an environment. Let's try to describe these specifics:

1 For most classes are using the same software configuration of work stations (package Office + software specific to the various classes as Mathematica, Visual Studio package, graphics packages, etc.)

2 For some of the more advanced subjects a specific configuration is needed (usually requiring a more powerful computers) mainly in the case of configurations where the database is locally installed. Mostly these are the classes related to databases, ERP software (eg SAP with local database or computer networks (another network configuration or other elements of the local virtualization). This situation often is solved by separating the specialized laboratories or the ability to run different systems during take-off of systems (separate systems with different configuration on different disk partitions)

3 It is desirable that every student joining the course has a "clean machine " with no files or configuration changes that could leave the previous student

4 The key principle is that all the computers in the lab have exactly the same software configuration

5 One of the main problem of the administrators are configuration changes caused by students. The complexity of Windows causes that despite imposing further restrictions on student accounts, receiving permission to install there are still a lot of gaps, that clever students use to show their abilities (eg changing of desktop background, install add-ons to the browser, vulnerability installation malware). On the other hand, revoking causes problems with the software update, drivers (eg drivers for USB sticks), which handicap the life of students and teachers.

6 Most students perform simple tasks (eg working in Excel) by which the CPU utilization remains at $5-10 \%$ for $90 \%$ of the time.

So we summarize some of the implications arising from these observations:

Very time consuming and tedious for administrators is to maintain a number of such labs (in the form of a PC) and the continuous outgoing students of ingenuity who more or less deliberately modify the standard configurations of the operating system or application. Of course, this time consumption expresses the amount of administrators posts, who spend half of their time to perform the same, often unnecessary tasks. Of course there are administrative tools which allow you to automate part of the action (Active Directory, automation software installation and other), but here are new difficulties arising:

- not always administrators working on Colleges are proficient in implementing of new solutions (which implicitly from the earnings on such positions in the Colleges)

- tools to automate software deployment often require administrators to take the time to be able to perform this process. (not all programs can be automatically installed or require a rewrite to version installation "msi"

In addition, there are a number of activities which cannot be automated and require intervention as system recovery, repair damaged units etc.

We also need to take a minute to analyze the second point of described characteristic. Both of described variants are inefficient and cumbersome in practice. Creating specialized laboratories causes the difficulties in allocating and scheduling classes and must lead to not optimal time 
management of use of these laboratories or problems with their availability, especially if there are more. Often used on colleges the second option (multi - system configurations) is more efficient but requires a restart of computer before classes which practically can take up to 5 minutes and then require another restart (at the end or at the beginning of successive classes).

\section{ECONOMY BENEFITS OF A VDI IMPLEMENTATION IN STUDENT'S LABS}

From the analysis in the previous section is emerging organizational model of the optimal solution: ideally it would be if the student at the beginning of classes receives a computer with a freshly installed operating system and needed him during the classes applications and could do on it what he wants (and even in some cases have administrative privileges) and after completing the course, such a system were completely erased and in its place would be substituted entirely new.

Exactly this possibility gives the replacement of traditional PC by VDI architecture and terminal devices class "zero client".

However, all these considerations are only an introduction to change of the organizational model of maintaining laboratories, further we focus on the economic benefits and we will show savings that will allow institutions to manage more effectively its budget in the IT area :

- Expenses of maintaining administration - the implementation of the VDI architecture can significantly simplify the process of administration and maintaining laboratories and0 computer workstations. In the corporate environment in the 90s were taking that one administrative post covered support of about 50 workstations. With the passage of time and the expanding range of tools to automate administrative processes today it is assumed that one administrator (1 full time post) sufficient to support about 500 or more workstations. However in academicals environment (especially in the state Colleges) because of different not essential reasons, that go beyond this elaboration, development of increasing employment is observed in this segment. VDI specification itself causes that whether you have 100 or 500 workstations - for maintenance of laboratories and implementation of demand from the leading $1 / 2$ of the post is enough. The method of its distribution and scheduling is already in the hands of the management of the institution.

- Operating costs - a typical terminal integrated with LED monitor made with "zero client" technology consumes in average $40-50 \mathrm{~W}$ of electricity which is 4 times less consumption of a typical workstation (which consumes about $200 \mathrm{~W}$ with the monitor). Of course the VDI infrastructure includes also a set of servers and disk array, so averaging the results for a typical example of ten 30 - bench laboratories we can assume electricity savings of $50 \%$

- Costs of equipment replacement - assumed average amortization time of workstation to be three years, and in the university practice this time is estimated at 5 years. VDI equipment manufacturers as one of the advantages of VDI indicate twice as long amortization period of VDI client compare to typical workstation. The key is the fact that the VDI terminal has not in itself any components that determine aging hardware. So there is no processor or graphics card that will be too slow, disc you can drive crash, ending RAM, etc.. All of these components are found in servers, so long as the terminal does not break down naturally in the aging process of electronics or display - no need to replace it. VDI terminals have no mechanical parts including the lack of fans, so MTBF indicators (Mean Time between Failures) is for them around 70000 hours which is a value more than twice that of a typical workstation (MTBF - 30000 hours).

It should be noted, that alone cost of VDI terminal is about $1 / 4$ cheaper than the average computer set to the lab.

\section{TECHNICAL BENEFITS OF A VDI IMPLEMENTATIONS}

Further description of the functionality is based on the author's experience with VMware Horizon View [16], however, Microsoft's [17] solution offers similar functionality. Of course solutions based on VMware is currently considered the most technologically advanced, however, keep in mind that this solution is most expensive and, if in addition the University has the ability of implementation licensed under the MSDN Academic Alliance [18] (or another program) Microsoft solution can significantly reduce licensing costs and thus the entire implementation.

Philosophy and VDI infrastructure give administrators of the University efficient and stable management environment for teaching laboratories, automating many processes and increasing the reliability of the entire solution. The main aspects that empower administrators :

Central deployment and maintenance of virtual systems the administrator prepares a single system image, so called " Gold Image " which will be available in read-only option then cloning of each image and creating a virtual system does not copy the entire image. The system reads the data from the golden image and all the changes that are implemented in the virtual system are stored in so called paintings " Linked Clone " This process is visualized in the figure below. We compared here the volume of disk space for five virtual desktops, providing that each prospective of the images saved $1 \mathrm{~GB}$ of data for its own needs, while the volume of the golden image is $20 \mathrm{~GB}$ (see fig. 1). Of course, space images "Linked Clone" will be automatically increased while writing new data until achieving maximum value (defined by the administrator) [3] 


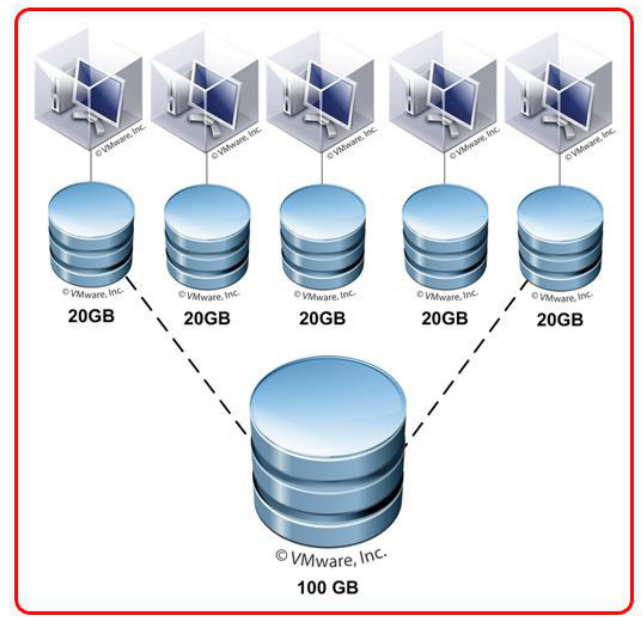

Classic allocation of virtual disks

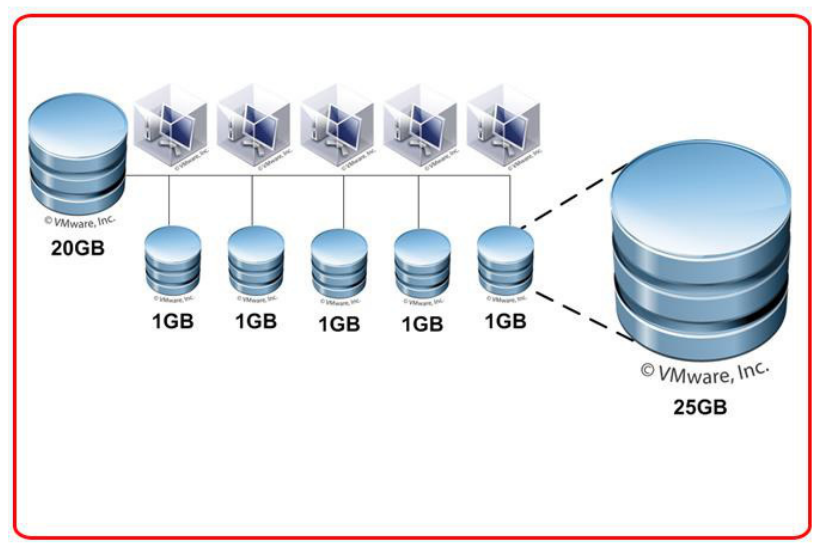

Technique "Gold Image" and "Linked Clone"

Fig 1. Compare disk space allocation.

For given example a space - saving (average) is $75 \%$ and in the process of increasing the number of working stations factor will still grow. Of course, this technique requires a sufficiently fast disk to store the golden image. It should be stored on disks SSD or disk data should be cached, preferably also using the SSD. Using such technology also gives you another key benefit for our case and laboratories namely fast refresh of virtual systems.

Technology "link cloned 'is particularly useful in these environments. We have here a lot in the exact same virtual machines allowing large savings relate the space of disk. For example, with 100 of the same virtual machine where each would take a 20GB and "link clone" would be at the level of savings we achieve $1 \mathrm{~GB}$ we can save almost 18 times more space on hard drives. (20GB x100 machines $=2 \mathrm{~TB}$ and 100 $\mathrm{x} 1 \mathrm{~GB}+20 \mathrm{~GB}=120 \mathrm{~GB}$ ). Estimate the space required (for similar parameters 20GB - used space and 1GB of "link cloned" is shown in Figure 2. The figure shows estimated disk storage capacity requirements (in GB) depending on the amount of the same virtual systems.

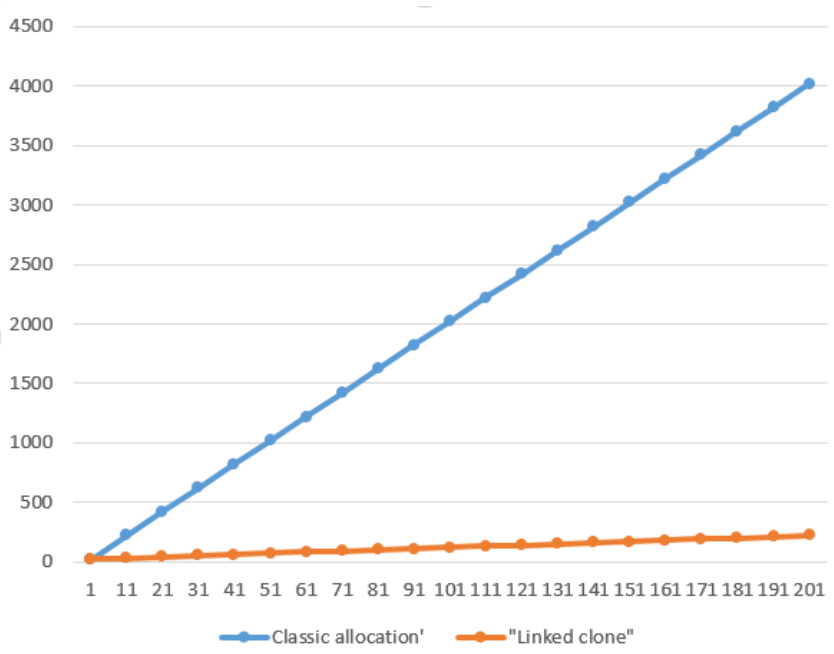

Fig 2. Estimated space required for virtual systems (in GB)

Instant Refreshing virtual systems - one of the implications of the golden images is the fact that if a virtual operating system only reads data from the golden image (without the possibility of writing anything on it) and all the changes differential writes on "Linked Clone", is to delete the data in this place immediately restore the clean image of the system (see fig. 3). The system automatically disconnects the user after 15 minutes of inactivity and instantly refreshes the image of the virtual system, which restores it to its original state (this process takes about five seconds for each system) [6][4].

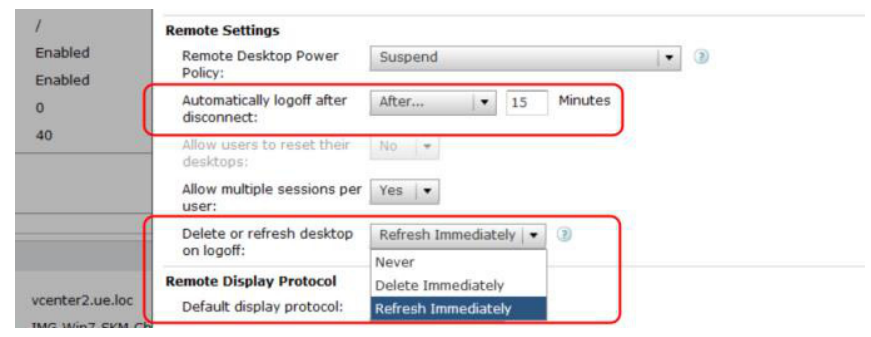

Fig 3. Refreshing virtual OS configuration

Business continuity of laboratory - User cannot spoil anything - unless he physically damages the terminal. Since the virtual system which works will be cleared immediately after work no matter how much he can reconfigure it, disorder or virused by student.

Security policy - depending on the philosophy adopted by the security administrators can be installed one central antivirus program with agents for individual virtual systems, or even resign from anti-virus software on workstations based on the assumption that even if the student hacked the computer, or even computers in the vicinity of this, anyway they will not exist after classes.

In addition, the VDI environment offers many useful additional features that allow you to: load balancing (moving virtual systems to the other servers in the cluster), shutdown of servers with lighter loads snapshots and much more.

Prior to the implementation, it is necessary, of course to 
ensure the layout of server resources necessary to ensure adequate performance - the next section describes an example configuration and load up to 300 images, on pages could be found relevant calculators to calculate the parameters of the environment.

\section{VIII.THE LIMITATION OF VDI TECHNOLOGY}

Although the systems used for laboratories are not especially graphically demanding (not taking in consideration specific graphics applications) you should be aware that the real-time transmission of multiple video streams can cause performance problems of the entire ecosystem of VDI. These problems are growing not only with an increase of the amount of terminals but also with an increase in resolution of displayed images (which begins to be particularly important in Full HD resolutions and larger).

The specificity of VDI[12] and streaming overshadowing many virtual desktops through a common internet network, the servers even when you start Youtube are very heavily loaded for two reasons:

FIRST REASON - PCoIP stream is encoded in the default workstation and sent to the LAN. While decoding the entire image (especially for the Full HD resolution and larger), it turns out that the process of decoding the stream can take as many as 50-60 \% of the two- core virtual processor. To remedy this server can be equipped with a special card decoding hardware PCoIP streams. (Card Teradici Apex 2800 [15]. Application card offloads streaming process several times. One card is able to decode at the same time 40 HD streams or 25 streams of $2560 \times 1600$.

SECOND REASON - problem with such high resolutions: default graphics 2/3D is emulated by VMware as for the desktop becomes a bottleneck (especially using newer Windows systems, even with off AERO). The solution to this problem is the use of specialized graphics cards designed for VDI environments - (eg Nvidia Nvidia Grid K1 or K2 Grid)[13] which can be used SVGA (shared VGA) graphics card that is shared for multiple virtual systems. Although the power of the card (and the price as well) seems to be huge in the case of dividing its power for 20-30 virtual machines, can barely support the basic graphical operations.

- Both of the above measures cannot be applied in Blade servers, though the HP Blade Gen8 solutions it is possible to use a miniaturized version of the card. Projecting such solutions for large format displays, should mount them on traditional servers (like dell R720) [14].

\section{EXAMPLES OF IMPLEMENTATIONS OF VDI IN UNIVERSITY OF ECONOMY IN WROCLAW}

The concept of rebuilding IT Infrastructure on University of Economics and building its own private cloud began to emerge in mid-2010 and after passaging of all the procedures, fixing concept and finding financing of the project, in September 2011 begin the process of public procurement and project was run in early 2012. The University of Economics was the first university in Poland, which has implemented such solutions on such a large scale.

On the university are working more than 240 terminals (mainly Samsung NC240), more than 400 virtual systems and students can connect to one of 5 available images, depending on the classes and necessary configuration. For the purposes of VDI six 2-processor servers are dedicated, giving a total of 1.2 TB of RAM. Disk array and some servers are equipped with cache memory for SSD -based. One of the servers is equipped with an nVidia card GRID K1 and streaming card supporting hardware PCoIP (Apex 2800).

As storage is used EMC disk array with a total of $20 \mathrm{~Tb}$ working gross capacity.

\section{X.ANALYSIS OF SYSTEM PERFORMANCE VDI IN PRACTICAL EXAMPLE}

Instead of carrying out theoretical calculations functioning VDI performance will show a practical example of the above-described example, the our University (University of Economy in Wroclaw).

As we mentioned we use a few images of virtual system and the main virtual computers are:

1. Windows XP - with $1,5 \mathrm{~Gb}$ RAM and one core processor, $60 \mathrm{~Gb}$ provisioned disk and emulated graphics card by vmWare system. Most uses for office and economics applications.

2. Windows XP with Oracle local database - with $2 \mathrm{~GB}$ RAM, two cores, 40Gb provisioned disk, shared graphics card like Nvidia Grid K1 and PcoIP accretion card (Apex 2800). This system is used for classes of databases

3. Windows 7 - with $3 \mathrm{~Gb}$ RAM, two cores, 50Gb provisioned disk and shared graphics shared graphics card like Nvidia Grid K1 and PcoIP accretion card (Apex 2800). This system has software for computer networks and some graphics and business applications.

Below we present some examples of server load these virtual systems in a typical working day. The fig. 4 shows typical load the entire cluster. The cluster consists of:

- Five two-processors Xeon E5645 - 2,4GHz (16 logical cores) servers (Dell M710HD) with 196Gb RAM each -described as ESX2 - ESX6 on the figs.

- One two-processors Xeon E5-2630 - 2,3GHz (24 logical cores) server (Dell R720) with 262Gb RAM each, with NVIdia Grid K1 Video Card and Hardware Acceleration PcoIP Card (Apex 2800) -described as ESX17 on the figs. 


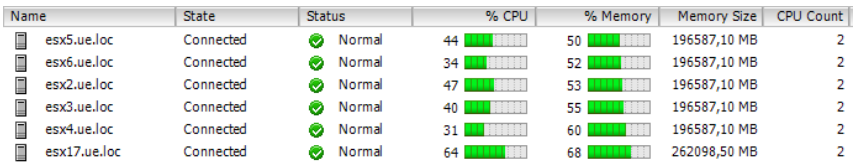

Fig 4. Typically loaded VDI cluster

On the ESX17 there is 84 virtual systems:

- 32 virtual systems of Windows XP with Oracle local database

- 52 virtual systems of Windows 7

On the fig. 5 and 6 we can see typically load virtual systems when the student class working.

\begin{tabular}{|c|c|c|c|c|c|c|c|c|c|c|}
\hline \multicolumn{2}{|c|}{ Name } & State & Statu & & | Host & Provisione & Used Spat & ost & Host Mem-1 & vest Mem -\% \\
\hline & Win7-VS2013-9 & Powered On & 0 & Normal & ess3.ue.loc & $80,78 \mathrm{~GB}$ & 30,78 GB & 0 & $2781=$ & 2 \\
\hline & Win7-V52013-8 & Powered on & - & Normal & esx5.ue.loc & 80,78 GB & $30,78 \mathrm{~GB}$ & 0 & $2761=$ & \\
\hline E & Win7-VS2013-7 & Powered on & - & Normal & esxs.ue.loc & 80,78 GB & 30,78 GB & 0 & $2759=$ & $3 \square$ \\
\hline 40 & Win7-VS2013-6 & Powered on & 0 & Normal & esx3.ue.loc & $80,78 G B$ & 30,78 GB & 231 & $2938=$ & 1 \\
\hline 胥 & Win7-vS2013-5 & Powered on & $\bullet$ & Normal & esx6.ue.loc & $80,78 \mathrm{~GB}$ & $30,82 \mathrm{~GB}$ & & $1166=$ & $3 \pi$ \\
\hline 80 & Win7-vS2013-4 & Powered on & 0 & Normal & esx2.ve.loc & & 30,78 & 231 & $2756=$ & \\
\hline & Win7-vS2013-35 & Powered on & 0 & Normal & esx3.ue.loc & & & & $1230=$ & \\
\hline & Wint-v & Powered on & 0 & Normal & esx2.ve.Joc & & & 471 & $2782=$ & \\
\hline & Win 7 vs & Powered on & 0 & Normal & esx6.ue.Joc & & 3 & 231 & $788=$ & \\
\hline & Win7-vs & red on & 0 & Normal & esx2.ve.loc & $80,78 \mathrm{~GB}$ & $0,78 \mathrm{~GB}$ & 95 & $768=$ & \\
\hline in & Win7-vs2 & Powered on & 0 & Normal & esx4.ve.loc & $80,78 \mathrm{~GB}$ & $30,78 \mathrm{~GB}$ & 95 & $2776=$ & \\
\hline 褁 & Win7-V52013 & Powered on & 0 & Normal & esx3.ve.loc & 80,78 GB & 30,78 GB & 711 & $2925=$ & \\
\hline 跑 & Win7-VS2013-3 & Powered on & 0 & Normal & esxa.vel.joc & 80,78 GB & $30,78 \mathrm{~GB}$ & 951 & $2796=$ & \\
\hline [8 & Win7-v52013-29 & Powered on & 0 & Normal & esx3.vel.loc & 80,78 GB & 30,78 GB & 711 & $2788=$ & \\
\hline D & Win7-v52013-28 & Powered On & 0 & Normal & esx5.vev.loc & 80,78 GB & 30,78 GB & 711 & $2768=$ & \\
\hline
\end{tabular}

Fig 5. Typically loaded Windows 7

1 Day Summary for esx17.ue.loc

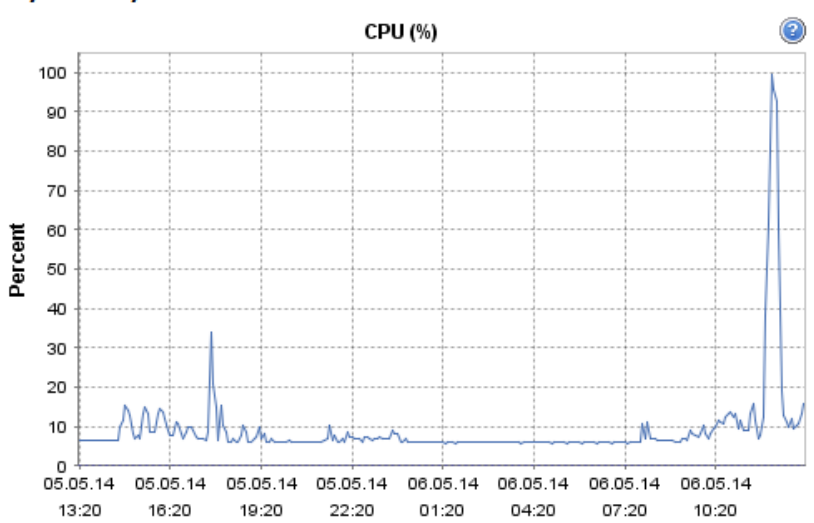

Fig 6. Typically loaded CPUs at ESX7 at worked day

On the ESX2- ESX6 we have 84 virtual systems:

- 32 virtual systems of Windows XP with Oracle local database

- 52 virtual systems of Windows 7

On the fig. 7 we can see typically load virtual systems with windows XP.

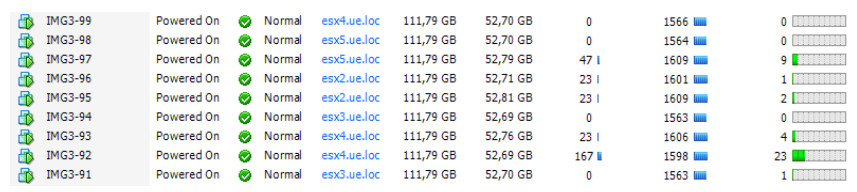

Fig 6. Typically loaded Windows XP with emulated graphics cards
Of course the Windows XP is the best system for virtualization because of low demand for computing power and RAM size as we can see on fig. 7.

\section{CONCLUSION}

After 2 years from implementing VDI on owner University (UE in Wroclaw) we know that VDI promises more efficient use of the university's resources, and could offer students the convenience of accessing specialty software from any device, at any time, from anywhere. Now we do not provide access from anywhere for students (just for teachers and some students involved in student's organization) but I's the next planned step.

\section{ACKNOWLEDGMENT}

Special thanks to other employees University of Economy in Wroclaw (administrators and the IT department of managers, employees of the Institute of Business Informatics, IT staff procurement and other departments involved in the project implementation and technology private cloud vdi on our University.

\section{KEYWORDS}

VDI, Virtual Desktop Infrastructure, Virtualization, Academic Computing, Private Cloud, Management academic laboratories

\section{REFERENCES}

[1] Serafin M., 2011, Wirtualizacja w Praktyce, Helion, Warszawa.

[2] Finn A., Luescher M., Lownds P., 2012, Windows Server 2012 Hyper-V. Podręcznik instalacji i konfiguracji, Helion, Warszawa.

[3] Lowe S.,Marshall N., 2013, Mastering VMware vSphere 5.5, John Wiley \& Sons, Indianapilis, Indiana

[4] Guthrie F., Lowe S., Coleman K., 2013, VMware vSphere design, John Wiley \& Sons, Indianapilis, Indiana

[5] Rosenberg J., Mateos A.,2012, Chmura obliczeniowa. Rozwiąania dla biznesu, Helion, Warszawa

[6] Asselin S., O'DohertyP., 2014, VMware Horizon Suite: Building End User Services, VMware Press

[7] Madden B., Knuth G., 2014, Desktops as a Service: Everything You Need to Know About DaaS \& Hosted VDI, Burning Troll Production, San Francisco, California

[8] Miller K., Pegah M.,Virtualization, Virtually at the Desktop

[9] Vieira S., Why Virtual Desktop at CCRI ? Finding Sustainability for Desktop Support 
[10] Eaves A., Stockman M., 2012, Desktop as a Service Proof of Concept, 13th annual conference on Information technology education, pp. 85-86, ACM New York, USA

[11] What are the benefits of VDI?

http://www.techrepublic.com/blog/the-enterprise-cloud/whatare-the-benefits-of-vdi/579/

[12] Wady i zalety wirtualizacji stacji roboczych http://www.itwadministracji.pl/numery/marzec-2014/wady-izalety-wirtualizacji-stacji-roboczych.html

[13] GRID GPUS http://www.nvidia.com/object/grid-boards.html

[14] GPU accelerators and coprocessors for PowerEdge servers http://www.dell.com/learn/us/en/04/campaigns/poweredgegpu
[15] Accelerate the Virtual Workspace: Take a Load off Servers http://www.teradici.com/products-and-solutions/pcoip-products/hardware-accelerator

[16] VMware Horizon View http://www.vmware.com/pl/products/horizon-view

[17] Microsoft Virtual Desktop Infrastructure (VDI) http://www.microsoft.com/pl-pl/windows/enterprise/productsand-technologies/virtualization/vdi.aspx

[18] Microsoft DreamSpark https://www.dreamspark.com/

[19] VMware Infrastructure VDI Server sizing and scaling http://www.vmware.com/pdf/vdi_sizing_vi3.pdf 